\title{
Supporting Worth Mapping with Sentence Completion
}

\author{
Gilbert Cockton $^{1}$, Sari Kujala ${ }^{2}$, Piia Nurkka ${ }^{2}$, and Taneli Hölttä ${ }^{3}$ \\ ${ }^{1}$ University of Sunderland, Faculty of Applied Sciences, Sunderland, SR6 0DD, UK \\ ${ }^{2}$ Tampere University of Technology, P.O. Box 589, FI-33101 Tampere, Finland \\ ${ }^{3}$ Paf, Unioninkatu 24, FI-00130 Helsinki, Finland
}

\begin{abstract}
Expectations for design and evaluation approaches are set by the development practices within which they are used. Worth-Centred Development (WCD) seeks to both shape and fit such practices. We report a study that combined two WCD approaches. Sentence completion gathered credible quantitative data on user values, which were used to identify relevant values and aversions of two player groups for an on-line gambling site. These values provided human value elements for a complementary WCD approach of worth mapping. Initial worth maps were extended in three workshops, which focused on outcomes and user experiences that could be better addressed in the current product and associated marketing materials. We describe how worth maps were prepared for, and presented in, workshops, and how product owners and associated business roles evaluated the combination of WCD approaches. Based on our experiences, we offer practical advice on this combinination.
\end{abstract}

Keywords: User Values, Sentence Completion, Worth-Centred Development (WCD), Worth Mapping, Monetary Gaming.

\section{Introduction: Holistic Worth-Centred Interaction Design}

HCI needs to develop more holistic design practices, i.e., combinations of approaches that give equal attention to all aspects of design and evaluation and work well together. olistic practices balance attention to choices of means, ends, beneficiaries and evaluations [1], but more importantly create and maintain synergies between and across them. This focus on synergies, or links between choices of means, ends, beneficiaries and evaluations, results in designing as connecting [1], rather than designing as crafting. Design choices extend beyond choices of means (e.g., features) to choices of ends, stakeholders ('beneficiaries') and validations ('evaluations'). By covering the classes of design choice, and their inter-connections, holistic design practices are supported by a wider frame of reference for designing.

Figure 1 shows six connections between four classes of design choice. The three dotted connections associated with evaluations are not in scope for this paper. Of the remaining two connections, the lowest one between means and ends is the main focus of worth maps [1], which are networks of elements and associations that contain means-end chains that hopefully causally link design elements to human elements [1]. Solid connections from beneficiaries to both means and ends are the main focus for a sentence completion approach. These two WCD approaches interface via a design's ends, that is, the explicit purposes of a design. Sentence completion provides direct 


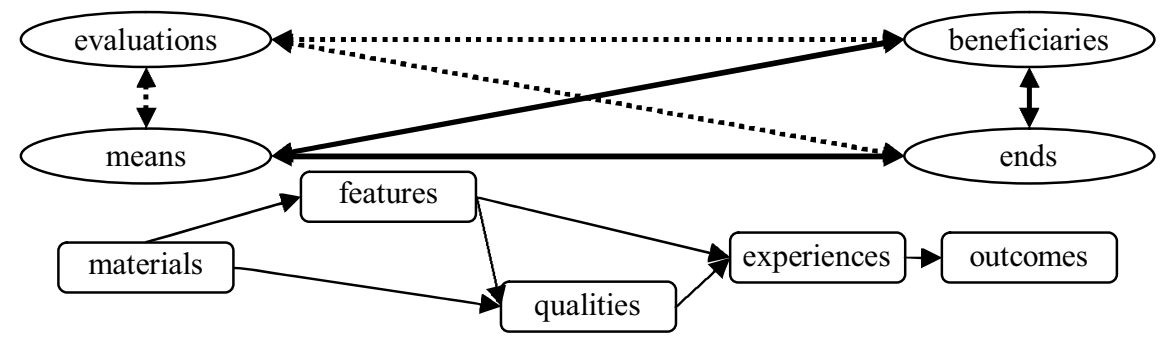

Fig. 1. Designing as Connecting, with means-end chain of worth map elements below

access to people's values in specific usage contexts, which values can be mapped onto outcome elements in worth maps. Sentence completion provides user data on benefits (a.k.a. value/s) that motivate users, and on the costs (perhaps adverse) that demotivate them. Once costs and benefits are identified, likely balances of worth can be assessed.

Sentence completion and worth mapping are called 'approaches', rather than 'methods' or 'techniques' because we cannot rigidly direct human activities (especially creative ones such as design). Instead, approaches combine flexible representations with suggested modes of use, theoretical and practical knowledge, perspectives, insights, and guiding values. Approaches leave project teams to find their own way through product or service development.

Worth maps have already been used in a range of explorations. They are network diagrams that use lines to indicate associations between human and design elements. These associations build vertically into means-end chains (an abstract one runs horizontally across the bottom of Figure 1, (see Figure 3 and [3] for example worth map structures). Worth maps explicitly connect design means with design ends, indicating 'why' people would (not) use a system. User Experience (UX) elements are the interactive means that link design ends (outcomes) with design means (qualities, features, materials). Good UXs satisfy motives.. Sets of worth maps can visualize a complete product context [2].

As with all approaches within a WCD framework, worth maps only support a subset of six design meta-principles [1]. Relationships between WCD approaches and meta-principles are important, since the latter scope the former. An approach needs to be evaluated on the bases of the meta-principles for which it provides support. Worth maps primarily support the committedness meta-principle through a single representation that can reference a project team's current set of choices of means, ends, beneficiaries, evaluations and their inter-connections. Worth maps also support expressivit, through visual representations of means-end chains. However, they do not support receptiveness, which requires openness to alternative design means, ends, evaluations, and a range of beneficiaries. Sentence completion can thus complement worth maps through receptiveness to design ends as user values. By providing data and analyses to identify (un)desirable design ends (outcomes), sentence completion also supports credibility by grounding design purpose (ends) in inspectable user data. 


\section{Research Instruments for User Values}

Values are abstract constructs that cannot be directly observed. People can talk about them, or they can be inferred from observations of people, places and/or things. However Hoyer and MacInnis [4], note that people do not often think about their values and can not easily verbalise what is really important to them. This presents different research challenges to understanding users' activities, which has been the dominant focus in HCI, although this is not to say that such observational studies are straightforward and without challenge. Even so, observations may not be the most effective research instrument when trying to access user values.

Some research instruments directly access a pre-determined context-free set of values, but these questionnaires make assumptions about values (e.g., the Rokeach RVS [5] and Schwartz SVS [6] value surveys). However, people can value something without being able to associate motives with pre-identified named values. Feelings tell people what is (not) worthwhile, but without always exposing value drivers. Thus validated psychometric instruments may limit accessible user values, perhaps to either too narrow a range of values, too abstract a set of values, or both.

Projective research instruments are more open than closed value surveys: examples include word association tests, sentence completion, drawing, writing and story completion [7]. These elicit qualitative data that people cannot or will not verbalize via more direct instruments. Many were developed in therapeutic or child research settings with limited verbal fluency and/or difficult access to feelings. Projective instruments elicit indirect responses, either to ambiguous stimuli or talking about objects, situations, or other people's feelings, attitudes and opinions. Presented stimuli are very ambiguous; individuals' responses can reveal fundamental modes of thinking and behaving. Also, in talking about a third party or object, respondents may project covert feelings, which may be subsequently discussed [7]. Banister and Booth [8] experimented with different projective techniques to explore innovative methodologies for child-centric consumer research. In drawing tasks, they provided templates blank except for an outline. Given a tree outline, children were asked to decorate it as a tree of disgusts, tree of not very good taste, and tree of very good taste. This prompted insightful data on likes and dislikes without being intrusive, with children's experiences communicated through their drawings.

Projective techniques are now more common in HCI [10]. Cultural probes aim to stimulate participant reactions [9] through a probe pack of artefacts for use in directed activities, such as disposable cameras for taking photos, or a voice recorder for capturing dreams. Cultural probes took advantage of rapidly falling prices of recording devices and for custom printing, extending the range of respondent activities with projective instruments. Voida and Mynatt [11] modified cultural probes and combined them with RVS [5] to elicit values from two families. Their value probes directed families to complete several value manifestations, for example, a Family Album (how they portray themselves), a Day Planner (how they spend their time, actually then ideally), a Map (how they use their space), a Budget (how they spend their money), and a Scrapbook (what they surround themselves with). Four student design teams each completed a RVS based on returned probes (two teams were assigned to a family). The teams' RVS results were then compared to their family's, with good matches at the extremes (most/least important values). However, while the RVS did prompt 
students to re-examine probes for manifestations of initially overlooked values, probes also manifested values that could not be associated with any RVS value ([11] p. 2013). This supports the claim that existing validated closed value surveys may not be comprehensive enough to connect with the tacit 'value' that can be provided by technological innovation.

Projective research instruments can thus complement observation, interviews and closed format questionnaires. However, an instrument's worth is inherently situational, and thus we cannot establish the relative worth of one instrument over another. There are no absolute orderings, with one approach inherently superior to another. As worth is situational, a project may not be able to afford extensive observations and/or triangulation interviews; or a project sponsor may prefer quantitative methods over interpretative methods that make inferences from informants' accounts of actions. Thus, for example, cultural probes have high production values, requiring highly creative design and selection of artefacts for the probe pack to ensure high aesthetic standards. Not all project teams can bear such costs. In addition, interpretation of returned probes should be creative and open, which again may be unsuitable for project milieux where cultural probes would be misused through systematic quantitative analysis [10].

\section{Using Sentence Completion to Elicit Values}

Our main case study sought to develop a user research approach to provide quantitative data on user values that could be affordably scaled to large samples and could integrate well with worth maps. We thus explored projective sentence completion as a research instrument for credibly identifying common user values.

\subsection{Background}

In sentence completion, a person is asked to complete incomplete sentences with their first reaction, since they are in a written form. Sentence completion is well established in consumer psychology. For example, Hoyer and MacInnis [4, p. 60] asked cigarette smokers why they smoked. Most said they enjoyed it and believed that smoking in moderation was fine. However, when given incomplete sentences like "People who never smoke are ___, they filled in the blanks with words like 'happier' and 'wiser'. And, given sentences like "Teenagers who smoke are ____, respondents answered with words like 'crazy' and 'foolish'. Smokers were clearly more concerned about smoking than their explicit answers indicated [4].

\subsection{Theoretical Support for Values Elicitation}

Sentence completion is a suitable questionnaire format for identifying user values. Unlike closed questionnaire formats (e.g., [12]), sentence completion tasks do not prime respondents with direct questions about specific emotions or values and their product or service associations. Sentence completion makes no assumptions about fixed sets of human values (as do RVS [5] and SVS [6]), but can still be guided by various literatures on human values, which we surveyed to identify ten categories of 
Table 1. Categories of Values for Receptiveness in Sentence Completion (based on [13])

\begin{tabular}{|c|c|}
\hline Category of value(s) & Examples \\
\hline $\begin{array}{l}\text { Social Relatedness } \\
{[5,6,12,14]}\end{array}$ & $\begin{array}{l}\text { Esteem, status, power, control and dominance, } \\
\text { achievement, conformity, equality, helpfulness, } \\
\text { honesty and loyalty }\end{array}$ \\
\hline $\begin{array}{l}\text { Emotional and hedonistic } \\
{[5,12,14]}\end{array}$ & $\begin{array}{l}\text { Aroused feelings or affective states, pleasure, fun, } \\
\text { sensory enjoyment }\end{array}$ \\
\hline $\begin{array}{l}\text { Stimulation and epistemic } \\
{[5,12,14]}\end{array}$ & $\begin{array}{l}\text { Excitement, experienced curiosity, novelty and gained } \\
\text { knowledge }\end{array}$ \\
\hline $\begin{array}{l}\text { Growth and self- } \\
\text { actualization }[5,14]\end{array}$ & $\begin{array}{l}\text { Independent thought and action: choosing, creating, } \\
\text { exploring }\end{array}$ \\
\hline $\begin{array}{l}\text { Traditional } \\
{[6,14]}\end{array}$ & $\begin{array}{l}\text { Respect, commitment, and acceptance of customs and } \\
\text { ideas that traditional culture/religion impose on the self }\end{array}$ \\
\hline $\begin{array}{l}\text { Safety }[12,14] \\
\text { Universal values } \\
{[12]}\end{array}$ & $\begin{array}{l}\text { Security, social order, health, comfort, free from fear } \\
\text { Understanding, appreciation, tolerance, and protection } \\
\text { for the welfare of all people and for nature }\end{array}$ \\
\hline
\end{tabular}

potential user value(s) from a broad range of theories of human motivation, social psychology and consumer behaviour.

Psychology and sociology have investigated the nature of values. For example, SVS [6] value categories have been shown to be valid in 21 countries. The relative importance of these categories constitutes an individual's system of value priorities. However, consumer behaviour and marketing identify different value categories, which correspond to unnamed concrete value, and not to named abstract values.

Table 1 summarizes six of ten value categories that we have identified, with sample source citations (for an extensive survey, see [13]). They are mostly based on psychological literature [5,6,14] or consumer psychology [12]. Although Maslow [14] writes about needs rather than values, we include these, reflecting extensive overlaps between concepts of not only needs, motivation and values, but also emotions and feelings. In Table 1, similar values are grouped into main categories. The most extensive is social; which values influencing people and relationships. Other categories relate to self, traditions and universal welfare.

There are two further categories of perceived value specific to human product relationships. Pura [15] mentions monetary and convenience value categories in her review. For monetary value, the product is seen as means of fulfilling tasks to derive monetary value. Convenience value gives a person ease and speed for achieving a task effectively and conveniently. Also, Boztepe [16] stresses the utility value of products alongside other value categories. A similar specific category, conditional value [12] only arises in a specific context, e.g., buying Christmas cards.

Knowledge of these ten categories of potential user and product related value(s) identified above can guide design of sentences for completion, illustrating the value of receptiveness to theories in WCD [1]. These categories can guide development of introductory sentences that probe general reactions to life and the focus topic, but are 
open enough to not prime responses. However, more focused sentences must probe specific value categories that are important to potential users. Before combining sentence completion with worth maps, we explored the former in a different setting.

\subsection{A Familiarisation Study in Sentence Completion}

A familiarisation study focused on user values towards exercise, which interested an industry collaborator in the Finnish VALU research project. Table 1 supported brainstorming to develop 50 incomplete sentences, which were pilot tested with a 41 year old man and two women aged 35 and 30, revealing different values towards exercising. The man did not like exercising, but instead shared children's joy as he coached football. One woman enjoyed the physical experience and associated relaxation of exercise. The other woman was more goal-oriented, trying to improve her performance. After this pilot, ten families were recruited to try out sentence completion alongside a familiar established research instrument, semi-structured interviews, with well documented advantages, insights, disadvantages and pitfalls in the research methods literature. The 50 incomplete sentences from the pilot test were reduced to 25. Table 2 shows examples of incomplete sentences and parental responses, translated from Finnish. We recruited selecting different sizes, gender/age compositions and types of family (e.g., single parent). Participants were aged 32-43 years, had 1-4 children, and were given movie tickets to compensate participation.

Accompanying interviews lasted from one to one and a half hours, with all but one at respondents' homes, with assistance from an industrial collaborator. Before each interview, family members were told about the research in general and the procedure, then one parent was asked to fill out the sentence completion task and a background information form. During interviews, we discussed the family's, and particularly each child's, ways to spend time and exercise. There were three main interview themes: family-related; parent-related and child-related questions.

Interviews were audio-recorded and transcribed. Values identified by parents were coded using Table 1 as a tool. For example, if a parent said that it is very important that her child has fun while exercising, this was coded as emotional and hedonistic. To control bias, two researchers independently analyzed results and counted the

Table 2. Examples of incomplete sentences and example parent sentence completions

\begin{tabular}{|c|c|c|}
\hline Category & Example incomplete sentences & Completion by a parent \\
\hline \multirow[t]{3}{*}{ General } & $\begin{array}{l}\text { The most important thing } \\
\text { to me is... }\end{array}$ & $\begin{array}{l}\ldots \text { the well-being of me and } \\
\text { my family. }\end{array}$ \\
\hline & My children exercise ... & $\begin{array}{l}\text {... irregularly, but willingly } \\
\text { when they have company. }\end{array}$ \\
\hline & $\begin{array}{l}\text { It is important in my children's } \\
\text { spare time activities that ... }\end{array}$ & $\begin{array}{l}\ldots \text { she enjoys it and it is good } \\
\text { for her health }\end{array}$ \\
\hline Social & $\begin{array}{l}\text { My children receive positive } \\
\text { attention in spare time } \\
\text { activities if ... }\end{array}$ & $\begin{array}{l}\text {... he participates or succeeds } \\
\text { e.g. meets a goal }\end{array}$ \\
\hline $\begin{array}{l}\text { Stimulation } \\
\text { /epistemic }\end{array}$ & $\begin{array}{l}\text { About my children's way of } \\
\text { spending time I want to know... }\end{array}$ & $\begin{array}{l}\ldots \text { she enjoys it and it is good } \\
\text { for her health. }\end{array}$ \\
\hline
\end{tabular}


values mentioned, both in sentence completion and the interviews. Counts were based on judgements, as interpretation was needed to associate phrases and values. Sentence completion provided slightly more instances of values and more detailed descriptions than interviews, with better foci across a range of value categories.

Sentence completion often uncovered values unreported in interviews. For example, one parent's sentence completion revealed that, although her child's friends were important in encouraging him to exercise or join in a certain hobby, sometimes they teased him, making him unsure and passive. However, during interview, she only mentioned teasing at a general level. Also, in sentence completion, nine parents mentioned well-being and health as an important to pursue, but only three mentioned well-being in interview, and none health. Sentence completion was thus slightly more receptive and also required less time to analyse and administer than interview data: each interview lasted from 60-90 minutes, while sentence completion took 10-15.

Sentence completion was focused, direct and relatively efficient in terms of design, administration and analysis. Its speed, straightforwardness and focus would benefit our main design context, where it would be adequate compensation for losing out on the creative insights and rich responses of cultural or value probes. Again, no absolute comparison is being made here. Rather, different values in different development contexts favour one approach over another. Thus use of sentence completion in a family exercise context indicated that the balance of benefits over costs would be adequate for our intended context, where quantitative methods were more acceptable over open, creative interpretative ones. The use of cultural probes in such contexts results in potential misuse through overriding the core principles that guided their invention [10]. From the perspective of development organisations and project sponsors, lower potential costs of sentence completion contribute to its worth.

We concluded that sentence completion was a very acceptable alternative to interviews, especially as once designed, sentence completions can be used with larger samples with far fewer additional skilled researchers (analysis costs will still rise, albeit less steeply than those for interviews). Additionally, online sentence completion surveys are less expensive to administer, easy to modify, generate fast results (usually with good response rates), with data easily exported into suitable formats for analysis.

\section{Combining Sentence Completion and Worth Mapping}

An industrial collaborator in the VALU research project on user values wanted to explore worth mapping, so we used sentence completion to elicit user values that could be associated with proposed new product attributes for online monetary gaming, now a very popular leisure activity worldwide. The partner, Paf, is a gaming association governed by public law, whose goal is to raise money for the public good by offering gaming to the public. Paf wants to know more about their customers and their deeper motivations to play online monetary games, and thus support further development of online gaming services, while avoiding adverse gambling outcomes in a responsible way. A further objective was to gather more details on players who are less active on Paf's site and compare them to more regular players. Research topics included e-commerce, online gaming behaviour, and selection of the online games and 
gaming sites. Most published research focuses on exploring pathological gambling (e.g., [17]): few addresses why unaddicted people play monetary games.

While the commercial context is online gaming, the research goal was to underpin worth mapping with sentence completion, leveraging its receptiveness to user views, and to credibly ground design ends in user data. The case study combined independent research and analysis with three collaborative workshops over three months. Two workshops focused on results of sentence completion, prepared initial worth maps, and decided how to present them for a third workshop focused wholly on worth mapping. Formative evaluation insights for the two approaches were gathered during the first two workshops, with a summative evaluation at the end of the third. As with the familiarisation study, close collaboration with an industrial partner with a strong commercial interest in results brought well motivated participants, with approach usage and evaluations going beyond 'academic' exercises.

\subsection{Sentence Completion in Support of Worth Mapping}

The participant sample was based on Paf's earlier player segmentation studies. It was the first study of its kind, and involved online monetary players in Finland. The two most interesting player groups to Paf were selected. They have differences in age range, income, residential area, and education definitions, as well as with - most importantly - various specific playing behaviours and preferences. Invited respondents were selected from Paf's customer database, based on gaming behaviour on Paf's site matching a segment profile. The participation incentive was a prize draw. Prizes were appropriate to interests for the segmentation description (Group 1, sports item; Group 2 , gift vouchers).. The first group included 8 less active players, and the second 34 . Note that, referring to the WCD meta-principle of inclusiveness [1], only values from two user groups were considered; business value was not systematically addressed through any receptiveness to Paf's product stakeholders.

40 sentences for completion were designed to meet case study objectives, using the approach in the earlier pilot study. Incomplete sentences (translated from Finnish) included: (a) "In my opinion, online monetary games__", (b) "An unpleasant gaming experience emerges__", and (c) "I find myself playing mostly games that_". 17 background questions were answered. Sentences were completed online using the Webropol service, since respondents played monetary games online.

Once online responses had been gathered, a basic quantitative analysis (SPSS t-test) revealed statistically significant differences between active and less active players. Less active players preferred playing online monetary games alone, and their regrets suggest aversion to losing more money than planned. Active players, on the other hand, seem to have fewer regrets and get over losses more quickly. ontent analysis was used to categorize open answers for each sentence completion. Many qualitative differences were found among the player groups, replicating existing customer segmentation studies, but also providing new and more detailed information about players, revealing that each group's gaming behaviours differed in many ways, with different motivations. A chart was created for each sentence completion, with percentages for frequency of responses. Some respondents mentioned several issues and others none, so bar totals could be either side of $100 \%$. Figure 2 shows response frequencies for completing "When playing online, I feel myself _". Bar length 
indicates frequencies, and can prioritize user values for feature review and innovation, as well contrast gaming behaviour and reasons to play online monetary games within different market segments. For example, in Figure 2, Group 1 players experience more normal, calm, or satisfied feelings when gaming online, while Group 2 players seem to feel very excited or a regretful idiot. Such differences could be explained by different expectations or playing behaviours and their experiential consequences. However, quantitative rankings and comparisons must be used in combination with qualitative data. Numbers can inform design priorities, but should not dictate them.

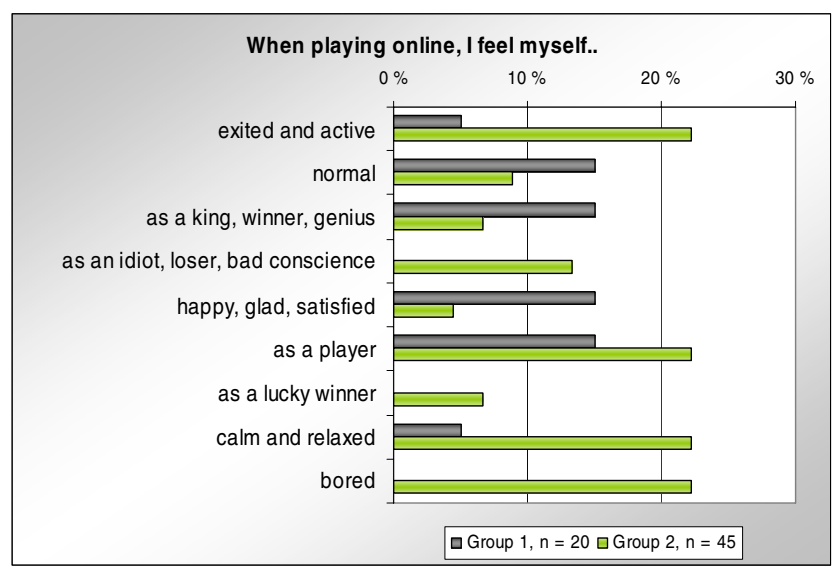

Fig. 2. Example Results of a Sentence Completion

Sentence completion was seen to support credible and expressive receptiveness to user values, although there were some later negative responses within Paf to using percentages with (for them) relatively small samples. Counts on horizontal scales of summative chart may be better. This aside, sentence completion via an existing web survey tool allowed relatively low cost collection of data

without researchers telephoning or visiting participants. Sentence completion results extended Paf's existing understanding of users' needs, motives, values, and especially aversions. This broader view of customer value called for new product features, but receptiveness to such features would have to be come from other approaches.

\subsection{Initial Worth Map Creation}

Figure 3 illustrates a worth map's structure, with a key to symbols on the left (Motives and Aversions respectively correspond to [3]'s Worthwhile and Adverse Outcomes). Upper and lower boxes (motives, UX, aversions) are human value elements that can be grounded in sentence completionI, indicating 'why' people would use a system (motives/benefits) or not use it (aversions/costs). Motives correspond to benefits, and aversions to costs. UX elements are the interactive means that link design ends (outcomes) with the design means (qualities, features, materials).

Worth maps support expressivity and credibility by explicitly connecting design elements and UXs to the underlying human motivations to (not) use them. A set of worth maps can visualize a complete product context, highlighting how user or supplier motives associate, via UXs, with design elements to enable (solid lines) or disable (dashed) positive or negative outcomes [2]. 


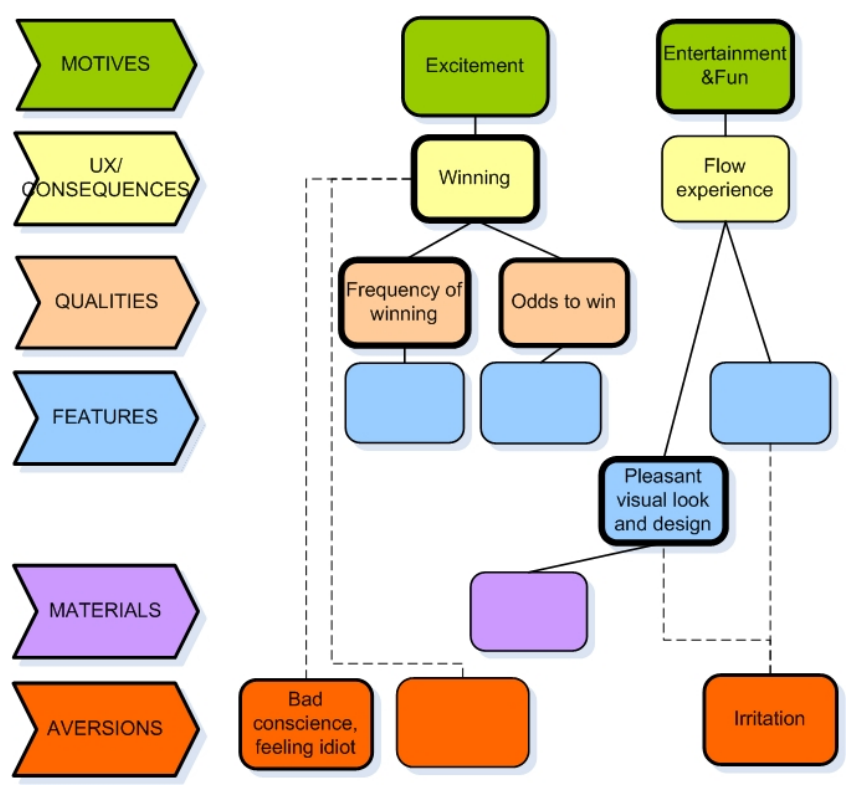

Fig. 3. Example Worth Map Layers

After researcher analysis and internal reporting of sentence completion results, two collaborative workshops further reviewed results and generated initial worth maps for each user group. Researchers and Paf representatives from User Experience and Player Intelligence roles attended. Sentence completion results were reviewed, and identified user values were transformed collaboratively into worth map elements, e.g., 'excitement' became a Motive element for both groups, and 'feeling loser and idiot' an Aversion element for Group 2. Sentence completion frequencies and existing player intelligence identified the most important issues for each group. Some sentence completion data covered design elements, which combined with existing ideas for new features to identify potential design elements to remove specific aversions or deliver specific UXs and their associated (connected) motivating meaning.

Several relevant product features were added to worth maps, connected to specific motives to play based on user data. To identify such connecting causalities between elements, answers of selected respondents in each group were examined question by question. Further new elements were added as they emerged. Elements were connected whenever associations were obvious. A guiding heuristic was that when respondents mentioned two consequent issues in their answers, these could be connected. Also, where workshop participants agreed on logically obvious - or otherwise known - associations (connections between elements), these were added, even without direct support from user data. The aim was to prepare partial worth maps for discussion, revision and extension in a third workshop. However, several identified worth elements were left with no associations connecting them to other map elements. To highlight these, and to aid map subsequent extensions, blank elements were added the partial worth maps to highlight the lack of associated product features. 
In [3], manual card sorting and arranging is proposed as a practical basis for worth mapping, where project teams gather around a large table to manipulate an evolving sketch or map. In this case study, a computer drawing tool was used. Once identified, each map element was added to a Microsoft Office Visio diagram, colour coded by category as in Figure 3 (thus creating a worth sketch [3] of unconnected elements). Worth maps were constructed for both player groups. Group 2's map had over 50 elements. Comments indexed by question and respondent number were added to elements to ground them in user data, e.g., " $22 \%$ of the respondents mentioned they feel excited and active after playing online" for the motive element 'Excitement'. Element boxe edges were given different line weights in Visio corresponding to frequency of mentions, thus highlighting potential high priority elements. This creative use of Visio extended the credibility and receptiveness of worth maps by directly and inspectably grounding human value elements in user data.

Another very effective innovation was a researcher's use of Visio drawing layers to modularise complex associations. All worth map elements were placed in the base layer, with each subsequent layer containing associations for means-end chains passing through a single UX element, making such chains easier to follow by hiding other layers. Element frequency coding, respondent comments, line weights, blank elements and means-end chain layers combined to improve worth map expressivity, and thus communicate them better to people not involved in the mapping process.

Figure 3 shows two layers for Group 2, showing means-end chains for the Winning and Flow UXs, which should lead to motivating outcomes of Excitement and Entertainment \& Fun. These UXs were thought to set requirements for the gaming site. To experience winning, frequency of winning and odds to win are key design qualities. Similarly, to experience flow, the site needs a pleasant visual look and design. No other design elements in support of positive UXs have been identified in Figure 3, hence blank elements draw attention to unsupported values and experiences. The 'pleasant visual look and design' element is a place holder for more extensive understandings of why existing layouts and sound may be inadequate. Worth maps support expressivity for connections between design choices, but not for choices themselves, which must be expressed by other approaches (e.g., Figure 2).

Identified aversions were also added as map elements, e.g., when not winning, but losing much and suffering a bad conscience, the excitement motive is not fulfilled. Similarly, irritating media usage will not lead to flow, entertainment and fun, as indicated by a dashed 'aversion block', indicating that a design element can help to avoid an adverse outcome. A dashed aversion block to 'irritation' requires subsequent close review of associations between multimedia features and usage outcomes. The extent of required creativity and sustained design management in WCD should not be underestimated. Design elements in initial worth maps are only possible means to achieve desired ends. Subsequent iterative design and evaluation is needed to show actual value achievement when users interact with new features. Figure 3 is thus one starting point for the third workshop. It is only one example of what worth mapping can achieve. The partial worth maps were extended further in the third workshop.

After two workshops and a familiarisation study, researchers and collaborators proceeded confidently to a third workshop, having seen sentence completion reveal what is (not) worthwhile for users. When presented as charts, results supported worth mapping, facilitating familiarity with data and staying anchored in it. However, with 
larger samples and sizable question sets, comprehensively walking through all data is too labour intensive. Data should be progressively sampled in reasonable chunks, with collaborative interpretation focused on qualitative insights and new product ideas. It is better to exploit sentence data in prioritised chunks, letting worth mapping proceed as collected data is being considered.

There was also early evidence that separation of design elements into materials, features and qualities was not helpful in this context. Such distinctions make sense to Interaction Designers and hardware and software engineers, but are largely irrelevant in high level mapping, especially when few developers or engineers are involved. Also, in the target web context, materials had little relevance (unlike in mobile and ubicomp settings). Hence materials were rarely used, and qualities and features were sometimes confused, e.g., 'a pleasant visual look and design' is both a quality ('pleasant') and a feature stub ('visual look and design'). Similarly, another map element, 'diversified, interesting games' (not in Figure 3), combined two qualities and a feature stub. A sensible appropriation here would have been to use a single unified product attribute element to avoid distraction until there was a real need to distinguish materials, features and qualities. Brainstorming could just identify 'design ideas'.

\subsection{Worth Map Review and Extension}

A key research objective of the third workshop was a summative evaluation of worth mapping. Paf's commercial goals were to share knowledge, understand how current services deliver identified customers' values, and how they can be improved (via brainstorming design ideas), as well as developing an understanding of worth maps' usefulness for Paf. Worth maps supported brainstorming on possible innovations for product design and marketing/customer relationship management (CRM).

The third workshop began with a 90 minute session before lunch that presented sentence completion results and introduced worth maps. In three hours after lunch, two teams reviewed, discussed and extended partial worth maps for each user segment, each time making a presentation to the other group. Each reviewed a worth map for both market segments of interest. Each team was facilitated by a researcher, with two Paf staff in each (overall, there were three product owners who had not taken part in previous workshops, plus a player intelligence analyst who had).

Understanding the worth maps was initially challenging, highlighting a need to explore ways of introducing prepared worth maps to people who had not constructed them. However, as participants came to understand how worth maps associate design features with user values, they were able to reassess existing ideas for product innovations, and also re-evaluate how products could be positioned in terms of a wider range of values, or to highlight alternative value propositions. Reconsidering existing proposed innovations within the context of worth maps did reframe how they were assessed. Also, ideas for additional design elements were proposed during discussions, spanning a wide range of design features from visual re-design, user control over some game attributes, and social computing. Again, the details of these features are not relevant to worth mapping, nor is their originality. Most additional features had already been identified in previous product review sessions before the worth mapping study. However, these features could now be closely associated with user motives and/or aversions. The credibility of such features changed, as did the extent of 
Table 3. Summative Evaluation Ratings

Question (some slightly reworded)

Av. (max 4)

1. Goals of mapping are understandable

2. Understanding user values is important in our business

3. Worth maps help to make user values, needs and motivations explicit

4. Worth maps help to design more successful products

5. After worth maps, I have a better conception of value of products/services

6. The worth map method is easy to use

7. Will use worth maps in the future

8. With worth maps, I can better connect product features with what really matters to users

9. With worth maps, I can better brainstorm

10. With worth maps, I can better develop a suitable marketing communications and manage customer relationships

11. With worth maps, I can estimate business value and use this in strategic decisions

12. Worth mapping results are easy to communicate further on

13. Worth maps suit company purposes well

14. Worth maps are useful in my work tasks

commitment to them by the project team. Features that were previously inadequately motivated could now be directly related to user costs and benefits. Worth mapping thus supported re-evaluation of existing or novel product features. Surprisingly, it also supported marketing and CRM innovations. Some marketing communication samples were briefly reviewed by each team, leading promisingly to new ideas about content and style. The lower relative cost of changing marketing communication materials meant that this could quickly translate into action.

Much of the above assessment is autobiographical. However, given the expectation that approaches are designed for appropriation, that is a key to any assessment of WCD frameworks. Thus innovative uses of Visio extended the expressivity of worth maps. In particular, use of layers avoided crowded, impenetrable diagrams in a way that is not possible with large single representation of 'table top' maps as advocated in [3]. We ended the third worth mapping sessions with a short participant questionnaire. The four commercial participants completed an evaluation form comprising 15 questions with a don't know option plus 4 point Likert scale (1=Strongly Disagree; 2=Disagree; 3=Agree; 4=Strongly Agree), followed by six open questions, which were not restricted to the scope of worth maps and sentence completion as understood in terms of WCD meta-principles. Thus despite low receptiveness to business value, we evaluated this and other 'out of scope' factors: Question 11 addresses 
inclusiveness of business value, and Question 4 addresses improvability. The results are shown in Table 3. As would be expected, the least favourable response is to Question 11. More inclusive use of maps needs (perhaps additional) user experiences, motives and aversions to have (additional) explicit associations with business beneficiaries. Business outcomes thus must be addressed separately from user outcomes, but must still be associated with them. Explicit direct receptiveness to business value would be required to improve responses to Question 11.

For success in practical settings, only means over 3 can be taken as good indicators of suitability for widespread use, which have been achieved for worth maps' main purpose of supporting designing as connecting ([1,3] Question 8) and committedness (Question 5). The mean score for Question 7 is a fair response overall, Improving this requires improvements on other responses, e.g., to Questions 3, 4, 6, 9, 12, 13 and 15, through use of approaches beyond worth maps and sentence completion, ideally making more use of existing Agile and relevant business approaches within Paf. Although almost 'good enough', brainstorming (Question 9) could be further improved by the use of other projective instruments reviewed above. Even so, the mildly positive responses on outcomes beyond the intended purposes of worth mapping and sentence completion are encouraging. Also, there was a fairly even distribution of positive and negative responses across roles, suggesting a lack of bias for product owners or marketing/CRM. Responses to open ended questions were mostly (very) positive, e.g., "Good tool to visually work with information", "good overview", "helps to connect abstract and concrete", "offering fine tuning and new products are found with it", "good for boosting workshops", "tool to brainstorm and to try to innovate new features", "possible to see [product] strengths and weaknesses". One participant preferred 'traditional' closed questionnaire formats to sentence completion, believing the former to be less leading, requiring less time for analysis and less researcher interpretation. However other participants valued sentence data as complementing existing marketing data, strongly indicating values. Three participants had not attended the first two workshops: one found worth maps difficult and confusing, especially the map element categories. Another found the density of connections challenging. Both could be avoided by further innovative use of drawing tools and improved workshop preparation and tutorial materials.

\subsection{Later Practical Utilisation of the Results}

Since the workshops, all project deliverables have been published via the company Intranet and Wiki. Three presentations, open to all interested personnel, were held: two on site in two of Paf's biggest office locations, and the third via overseas video conference. Following each on site presentation, a further workshop was held, where worth maps were walked through layer by layer, each showing a part of the whole map that was related to one of 4-6 'top issues', as identified through each player group's values. One workshop was more markets and sales focused, the other more product focused, in terms of participants and facilitation. In both, many evaluative notes were produced and discussed: some were evaluations of the existing offering; some were related 'design ideas' - some new, and some previously conceived but now further supported or differently anchored. Many ideas were quite high level (or epic!), which is in line with worth maps' expressivity, but some were specific. After 
the workshops, a collection of notes (as high resolution digital images), and a written summary of emergent topics were shared among the invitees. The third presentation drew most of all country organizations' management. Besides introducing all the project deliverables, a summary of emergent topics was briefly presented. It is now up to each business manager, product owner, and other key people to form their overview of the deliverables, and use this as a part of various 'knowledge building blocks' supporting their daily work. However, the investment of resources in follow up presentations and workshops indicates that worth mapping and sentence completion provided genuine business value to Paf. Of course, more work must be done to realise commercial value, and no payback can be assessed until new features and CRM/marketing are implemented, but there is clear competitive potential.

\section{Conclusions and Future Work}

The two combined WCD approaches can be assessed relative to the meta-principles that they aim to support. Worth maps aim to support committedness and expressivity, while sentence completion supports receptiveness, credibility and (through bar charts) expressivity. Committedness is the extent to which a project team has made explicit their commitment to design means, design ends, intended beneficiaries and evaluations. Receptiveness is indicated by openness to alternative design means and ends (i.e, user values), beneficiaries, and approaches to evaluation. Expressivity is assessed in terms of how well design approaches in use communicate design options and choices. Credibility is assessed in terms of the feasibility or groundedness of a design choice. Worth maps fared well on committedness (Question 8) but less well on expressiveness (Question 12), although there were some positive results (Question 3).

Both worth maps and sentence completion are WCD approaches that let project teams reflect on worth as the likely balance of costs (as adverse outcomes/aversions) and benefits (as worthwhile outcomes/motives). Both performed well here. There were very positive responses on understanding the goals of worth mapping (Question 1) and their ability to improve understandings of product or service value (Question 5). Sentence completion was evaluated indirectly, but had a role in positive responses on Questions 3 and 10. The two WCD approaches thus combined well to provide good bases for understanding user values. Sentence completion helped to elicit values that users often find difficult to articulate. It also surfaced experiences and emotions, and not just goals and needs, as well as aversions, which were particularly fruitful when brainstorming product improvements. Worth maps linked user values, needs and motivations explicitly to design elements. Explicit sentence completion data on user values and aversions lets worth maps be grounded in user viewpoints.

The practical efficiency and effectiveness of worth mapping was improved through the use of Visio, but further innovative tool support and tutorial materials could reduce the time taken to understand worth maps authored by others. This would better support combined industrial use of worth mapping and sentence completion to let project teams innovate and appropriate methodologically to deliver worth.

Simplifications are also possible, such as a single 'design idea' category to replace qualities, features and materials. This should make worth maps easier to produce, use and communicate to colleagues who were not involved in constructing them. Separate 
worth map elements for qualities, features and materials are better suited to $\mathrm{R} \& \mathrm{D}$ teams with a predominantly creative and technical focus (i.e., user experience researchers, interaction designers, software/ hardware engineers). Worth maps with only 'design idea', UX and outcome elements could be initially developed by project teams with mostly business roles (e.g., product or service owners, marketing and CRM specialists, brand strategists), for subsequent refinement of 'design ideas' by a more R\&D focused creative and technical team. Modifications to approaches and project practices should be able to make worth mapping more worthwhile, but even so, its first use without its inventor present did result in worthwhile outcomes.

\section{References}

[1] Cockton, G.: Getting There: Six Meta-Principles and Interaction Design. In: Proceedings of CHI 2009, pp. 2223-2232 (2009)

[2] Cockton, G.: Designing worth is worth designing. In: Proc. NordiCHI 2006, pp. 165-174 (2006)

[3] Cockton, G.: Designing Worth - Connecting Preferred Means to Desired Ends. Interactions 15(4), 54-57 (2008)

[4] Hoyer, W.D., MacInnis, D.J.: Consumer Behavior, Houghton Mifflin, USA (2007)

[5] Rokeach, M.: The Nature of Human Values. Free Press, New York (1973)

[6] Schwartz, S.H.: Universals in the content and structure of values: theoretical advances and empirical test in 20 countries. Adv. in Experimental Soc. Psych. 25, 1-65 (1992)

[7] Donoghue, S.: Projective techniques in consumer research. J. Family Ecology and Consumer Sciences 28, 47 (2000)

[8] Banister, E.N., Booth, G.J.: Exploring innovative methodologies for child-centric consumer research. Qualitative Market Research 8(2), 157-175 (2005)

[9] Gaver, B., Dunne, T., Pacenti, E.: Cultural probes. Interactions 6(1), 21-29 (1999)

[10] Boehner, K., Vertesi, J., Sengers, P., Dourish, P.: How HCI interprets the probes. In: Proc. CHI 2007, pp. 1077-1086 (2007)

[11] Voida, A., Mynatt, E.D.: Conveying user values between families and designers. In: CHI 2005 Extended Abstracts, pp. 2013-2016. ACM, New York (2005)

[12] Sheth, J., Newman, B., Gross, B.: Consumption Values and Market Choices, Theory, and Applications. South-Western Publishing, USA (1991)

[13] Kujala, S., Väänänen-Vainio-Mattila, K.: Value of information systems and products: Understanding the users' perspective and values. JITTA 9(4), 23-39 (2009)

[14] Maslow, A.: Motivation and personality. Harper and Row, New York (1970)

[15] Pura, M.: Linking perceived value and loyalty in location-based mobile services. Managing Service Quality 15(6), 509-538 (2005)

[16] Boztepe, S.: User Value: Competing theories and models. Int. J. Design 1(2), 57-65 (2007)

[17] Lee, C.-K., Lee, Y.-K., Bernhard, B.J., Yoon, Y.-S.: Segmenting casino gamblers by motivation: A cluster analysis of Korean gamblers. Tourist Management 20(5), 856-866 (2006) 